\title{
Problem-based structure for a teaching-learning sequence to overcome students' difficulties when learning about atomic spectra
}

\author{
Francisco Savall-Alemany ${ }^{*}$ \\ Department of General and Specific Didactics, University of Alicante, \\ Sant Vicent del Raspeig, 03690 Alicante, Spain \\ Jenaro Guisasola \\ Department of Applied Physics, Polytechnic University College of Donostia, \\ University of the Basque Country, 20018 San Sebastian, Spain
}

Sergio Rosa Cintas

University Institute of Physics Applied to Science and Technology and Department of General and Specific Didactics, University of Alicante, Sant Vicent del Raspeig, 03690 Alicante, Spain

Joaquín Martínez-Torregrosa

University Institute of Physics Applied to Science and Technology and Department of General and Specific Didactics, University of Alicante, Sant Vicent del Raspeig, 03690 Alicante, Spain

(Received 18 February 2019; published 24 October 2019)

\begin{abstract}
Research has highlighted difficulties experienced by students when studying quantum physics in introductory courses. In this paper, we present a teaching and learning sequence (TLS) aiming at introducing a quantum model of emission and absorption of radiation and we assess its impact on the students' learning about atomic spectra. The TLS has been designed based on the contributions from physics education research and it was implemented among high school seniors. A detailed description of the TLS is also presented accompanied by a description of how it is implemented. Based on a pre- and post-assessment questionnaire and student interviews, we conclude that students are able to successfully use the models to reason about relevant phenomena.
\end{abstract}

DOI: 10.1103/PhysRevPhysEducRes.15.020138

\section{INTRODUCTION}

Quantum physics is an important part of introductory physics courses at high school and university [1-5]. The study of quantum physics usually begins by analyzing the phenomena of emission and absorption of electromagnetic radiation. Actually, a recent analysis of secondary school quantum physics curricula of 15 different countries has shown that "discrete energy levels" and "interaction between light and matter" are two of the most commonly occurring items [6]. One of the introductory quantum physics goals is that students grasp a quantum model of emission and absorption of radiation in order to explain the emission and absorption spectra, in terms of both frequencies and spectral line intensities.

\footnotetext{
*pacosavall@gmail.com
}

Published by the American Physical Society under the terms of the Creative Commons Attribution 4.0 International license. Further distribution of this work must maintain attribution to the author(s) and the published article's title, journal citation, and DOI.
This goal is relevant for two reasons. On the one hand, explaining gas spectra helped to highlight the shortcomings of classical physics and to establish some initial quantum ideas, in addition to having currently numerous research and technological applications [7-9]. On the other hand, the introduction of quantum physics is an extraordinary opportunity for students to work explicitly on the construction and use of scientific models. By doing so, their way of reasoning comes closer to that of expert scientists. In fact, research has shown that modelbased reasoning is at the core of any scientific discipline [10]. Models are representations used by scientists to simplify complex phenomena, to reason, to visualize abstract entities, and to interpret and predict experimental results $[5,11-14]$.

Several studies have revealed persistent learning difficulties regarding spectra and energy quantization in introductory quantum physics courses. Sinarcas and Solbes [15] found that high school students had difficulties answering the question "How does the Bohr model explain discontinuous spectra?", since more than $50 \%$ of the students did not give any answer. Didis and Wang [16] interviewed 9 
university students who had taken a course in modern physics, focusing on quantization of radiation and energy and angular momentum quantization in the atom. They found that only 2 students had learned a scientifically correct model, while the remaining 7 students had difficulties related to the key idea that every transition is produced by the interaction between a single photon and a single atomic electron. By analyzing other phenomena related to energy quantization in atoms and radiation (not atomic spectra), Didis, Eryilmaz, and Erkoç [17] stated that, after following a whole course in modern physics, only $10 \%$ of the university students acquired a scientifically correct model. Likewise, research on the photoelectric effect had detected learning difficulties regarding energy quantization in radiation and in moving beyond the wave model [18].

In a previous paper [19], we established the key concepts $(\mathrm{KC})$ that any quantum model of emission and absorption of electromagnetic radiation should consider in order to explain gas spectra in quantum physics introductory courses (see Table I). Moreover, we identified the main difficulties that high school students, university physics students, and high school teachers had regarding this issue. Specifically, we observed the following:

- Students have difficulties with energy quantization in the atom (difficulties with $\mathrm{KC} 1$ ). They consider that an atom can have any amount of energy. This difficulty was also detected by Didis, Eryilmaz, and Erkoç [17]. We also found that some students and teachers identified the base state with the energy level of $0 \mathrm{eV}$. This difficulty has also been detected by Ivanjek et al. [20].

- Regarding the energy quantization in radiation (related to $\mathrm{KC} 2$ ), some students consider that a photon can be partially absorbed (difficulties with KC2.1). Some teachers and students attribute greater intensity to radiation that is made up of higher energy photons, regardless of the number of photons (difficulties with $\mathrm{KC} 2.3$ ).

- The interaction between atoms and radiation (related to KC3) leads to a higher percentage of incorrect answers and a greater diversity of difficulties. As Zollman et al. [3] and Ivanjek et al. [20] did, we detected that some students and teachers consider that emission and absorption of radiation can take place without an atom transition between two stationary states (difficulties with KC3.1). Likewise, some students and teachers relate the emitted radiation energy to only one of the states among which the atomic transition takes place (difficulties with KC3.1). Another difficulty denote that transitions from higher to lower energy stationary states take place directly to the base state (difficulties with KC3.2). This difficulty has also been detected by Ivanjek et al. [20]. In addition, we found a difficulty among both teachers and students that is not strictly connected with quantum physics: they do not apply the principle of energy conservation to the interaction between atoms and radiation, so that the energy transition in the atom may not coincide with the energy of the emitted or absorbed photon. Finally, it was detected that a significant proportion of the teachers' group wrongly state that atoms absorb more than one photon and make successive transitions to higher energy states up until they ionize (difficulties with KC3.4).

It should be emphasized that, although these KCs refer to the emission and absorption of radiation and they can be considered to be typical of quantum physics introductory

TABLE I. Key concepts that should be considered in any quantum model of emission and absorption of electromagnetic radiation to explain the gas spectra [19].

$\mathrm{KC} 1$ (atomic model). Energy is quantized in atoms.

KC2 (light model). Energy is quantized in radiation.

KC3 (emission and absorption model). Every transition is produced by the interaction between a single photon and a single atomic electron.
$\mathrm{KC} 1.1$. Atoms can only be found in stationary states, characterized by discrete energy values, in which they do not emit energy. Any energy change entails the transition of the atom from one stationary state to another.

KC2.1 Radiation is composed of photons, understood as indivisible quanta.

$\mathrm{KC} 2.2$ Each photon's energy is proportional to the radiation frequency.

$\mathrm{KC} 2.3$ The radiation intensity is proportional to the number and energy of photons that form it.

KC3.1 The frequency of the radiation emitted or absorbed by an atom is proportional to the energy difference between the states from and to which the transition takes place.

KC3.2 Transitions to lower energy states are random, both in relation to the final state and in relation to the moment they take place.

KC3.3 The spectral lines' intensity is proportional to the number of atomic transitions per unit of time giving rise to them.

KC3.4 For a group of atoms at the lower energy level, the absorption of a photon produces transitions from that state to an excited state. Transitions from an excited state to another higher energy state are possible but highly improbable. 
courses, research has found that some students' difficulties in advanced courses are related to them and that incorrect learning in introductory courses has a negative impact on learning in later courses [21].

Some researchers have made proposals for teaching introductory quantum physics, but a lack of results concerning the learning outcomes has also been noted [18]. In the specific case of atomic spectra, Zollman et al. [3] present a sequence of computer-assisted activities aiming at studying the spectra of several light sources. They state that this sequence of activities brought positive results concerning students' learning and attitudes. McKagan et al. [4] test 18 computer simulations from the physics education technology project [22] that include several key features to help students build mental models about quantum mechanics. Over the course as a whole, where simulations were used extensively, the researchers found high learning gains. However, the researchers also indicate that students have difficulties when using the acquired models to explain other phenomena not contained in the simulations. Ivanjek et al. [23] present a research-based tutorial for students to correctly explain the frequencies of the emission spectra. After putting it into practice, the students were capable of applying the acquired concepts and reasoning skills in cases that differ from any other used during the instruction and they significantly improved their understanding. Hoehn and Finkelstein [14] use computer simulations to teach some quantum phenomena and note an increase in students' abilities to work with models, although they also note that there are conceptual difficulties that they are unable to overcome.

The fact that the learning difficulties are detected after traditional instruction, plus the diversity of results provided after implementing the new proposals, led us to design a teaching-learning sequence (TLS) to improve students' learning. Our study expands the aims of the aforementioned investigations in several respects. On the one hand, the TLS that we present deals with emission and absorption of radiation in a general way. It establishes a model that can explain not only the spectra but also other phenomena such as the photoelectric effect, fluorescence, and phosphorescence. Moreover, it also shows how devices such as lasers or LEDs work and other quantum interactions such as the Compton effect or the Franck and Hertz experiment. On the other hand, although we only analyze the impact on students' learning about atomic spectra, we also include more features than the previous research by addressing both the frequency and intensity of spectral lines in emission spectra and the absorption spectra.

\section{CONTEXT OF THE STUDY}

The TLS presented in this paper was implemented in the last year (second year) of high school in Spain. High school seniors (17-18 years old) who take the physics course receive 4 hours of class per week throughout the school year. The course is particularly intended for students who wish to study science or engineering at university. The curriculum content of this physics course is like the curriculum offered on standard physics courses at American colleges. In relation to quantum physics, the Spanish high school curriculum [24] includes standard learning aspects about the phenomena that give rise to quantum theory such as "To relate the wavelength or frequency of the radiation absorbed or emitted by an atom to the energy of the atomic levels involved" and "To interpret simple spectra, relating them to the composition of matter." In addition, other aspects related to the limitations of classical physics, such as the photoelectric effect, and Heisenberg and Schrodinger's contributions, such as "The quantum behavior of the particles. The need for a more general model to explain their behavior: the wave function and its probabilistic interpretation" are also included. The part of the curriculum covering quantum physics, nuclear physics, and relativity provides approximately $30 \%$ of the subject grade in external assessment tests taken by students to gain admission to Spanish university for science and engineering degrees. For this reason, teachers consider it important that students grasp the appropriate knowledge concerning these topics.

By "modern physics," the official syllabus and textbooks mean the scientific developments that gave rise to relativistic physics and quantum physics at the beginning of the 20th century. Regarding "quantum physics," in the introductory courses it is usually considered that it "begins" with the explanation of the blackbody spectrum by Planck in 1900. This is the sense that we give to the term quantum physics in our work. However, historians often reference "old quantum theory" as the period between 1900 and 1927 (prior to Heisenberg and Schrodinger's developments). Research in physics education classifies the atomic models introduced during this period (e.g., the Bohr model or the Sommerfeld model) as "semiclassical" or "quasiclassical." Their study in advanced quantum physics courses is controversial, as they mix classical ideas with quantum concepts that later makes it difficult to learn more current models, such as the atom model with orbitals based on the resolution of the Schrodinger equation [25-27].

All students had taken one semester of classical physics (spring semester) and one semester of chemistry (fall semester) in the first year of high school. The chemistry syllabus includes the Bohr atomic model and the quantum atomic model. However, the students had not studied the quantum model of radiation based on the photon concept, essential in interpreting the spectra. During this chemistry course the de Broglie hypothesis, the uncertainty principle or the probabilistic interpretation of the Schrodinger equation are not introduced. In relation to the teaching 
of atomic models, the main objective of the chemistry semester is an introduction to the study of the periodic table and chemical bonds. Students learn to apply the "Moeller rule" to fill atomic orbitals, predict the position of atoms on the periodic table, and analyze the chemical bonds they will form.

\section{PROBLEM-BASED STRUCTURE OF THE TEACHING-LEARNING SEQUENCE}

In this section we describe first the theoretical tenets of the TLS, then the pedagogical approach, and finally the development of the TLS.

\section{A. Theoretical framework of the TLS}

Over the last decades physics education research has repeatedly shown that students have failed to understand the basic concepts in physics [28]. This has highlighted the need for new learning strategies to replace the transmissive teaching of scientific concepts. This has resulted in a series of proposals that, disregarding minor differences, basically agree that instruction involves the active participation of the learner in the construction of 'new' knowledge and that they will have to use their previous knowledge as the basis for this [29]. Thus, we might speak of the rise of a social-constructivism psychological paradigm for the teaching and learning of sciences which must integrate not only the different teaching strategies but also those contributions coming from other fields that have produced enriching elements for science teaching, such as epistemology and ontology of science. These theoretical elements of teaching and learning show that students' meaningful learning of concepts depends not only on conceptual understanding, but also on students' procedures and attitudes [30,31]. This view of learning supposes that students inquire in problems or scenario posed by the teacher. They must use scientific skills, such as hypotheses, designing experiments to test the hypotheses, etc., to solve the problem and to build new knowledge guided by the teacher.

\section{B. Pedagogical approach}

The idea that the teaching and learning of sciences should recreate the scientific work is an old one [32]. However, the proposals we are presenting differ from those attempts in that the inquiry is not limited to learning how to test principles and in that students are not regarded as autonomous scientific researchers. In our proposal the teacher has an essential role to pose the problems and to guide both their resolution and the learning process. For this reason we call the approach "problem based-structure of learning and teaching" (PBSLT) [33-38]. For the resolution of the problems, the class is divided into small working groups (3-4 students) that develop a preliminary inquiry, cooperate between them, and interact with the scientific community, represented by the teacher and the literature. Then, the students' teams will receive aid in terms of clarifying questions, reformulate the partial results obtained, extracting conclusions, etc.

In accord with the PBSLT, we design a TLS structured as a succession of problems. At the beginning of the teaching a core problem is raised, and the entire TLS is structured around it. Next, the teacher proposes activities to make the students aware that they are dealing with an important and interesting problem. The teacher then asks what steps would be logical to follow in order to solve it, so both students and teacher should devise a plan, a sequence of specific problems (or steps), aiming at figuring out the main problem. Therefore, students progressively grasp the problem and the entire plan, so they feel that they have played an important role in it $[33,38]$. Concepts and models are introduced on a tentative basis, as informed hypotheses that seek a solution to the specific problems being raised. These hypotheses have to be tested through pencil and paper problems, laboratory experiments, predicting new results and problems, or analyzing their consistency with the knowledge already acquired [35-38].

As a whole, guided problem-based TLS encourage students to explain and confront their ideas in a hypotheticaldeductive environment involving many episodes of argumentation, fundamental for learning both scientific knowledge and science procedures $[39,40]$. Students discuss and work out activities in their small group. Then, group answers are pooled (agilely) and analyzed by the students and the teacher, coming to a reasoned consensus. During group work, the teacher's role is to encourage and guide students, to question their answers, and to make them think about it or provide additional information if necessary. These teaching strategies have demonstrated a positive impact on students' learning at several educational levels in fields such as optics [41,42], electromagnetism $[36,43]$, astronomy [34], problem solving [33], or experimental chemistry work [44].

The design of the TLS includes an epistemological analysis of the content established in the curriculum and an analysis of the learning difficulties of the students regarding that content. The epistemological analysis has been performed for the period 1900-1927, considering the physics level in university and college. This analysis covers the problems, hypotheses, ideas, and experimental research that make it possible to identify the key aspects and the main difficulties that historically the scientific community had to overcome in order to establish the scientific models that we set as learning objectives [[45-48], among others]. With regard to the students' difficulties on the subject, a review of previous research has been conducted [49,50]. Moreover, preliminary research was conducted on the students' difficulties in relation to the objectives defined in the TLS [19]. 


\section{TLS design phases}

The main objective of the TLS is to establish a model that explains how matter emits or absorbs radiation. This is the problem posed to students at the beginning of the unit and it guides the entire inquiry process. Before studying quantum physics, students have studied the wave nature of light. However, they have not considered how it interacts with a material system on a microscopic scale. We begin, then, by asking them about different ways of producing light and about phenomena that occur when light interacts with matter. In class, we produce combustion, incandescence, fluorescence, and phosphorescence phenomena (or show them on video) and devices such as energy-saving light bulbs, LEDs, and lasers are used. A spectroscope is introduced to analyze the characteristics of the radiation emitted by the different phenomena and/or devices in detail. It can be constructed and calibrated by the students [51].

In this initial phase, we want students to become familiar with the phenomena that we will try to explain later, which is essential for achieving adequate learning [4,52]. Next, we ask students for a tentative plan or strategy (which is the sequence "index") that we could follow to progress in terms of inventing a mechanism to explain the emission and absorption of radiation by material systems. After a short debate between the students and the teacher we agree that we should start with the apparently simplest situation: the emission of light by a gas formed by atoms that are as simple as possible. Thus, we begin the research by asking students to develop a model that explains the visible spectrum of hydrogen. As a hypothesis, students use the classical model of emission and absorption of radiation: the periodic movement of the electron in the atom might generate an electromagnetic wave of the same frequency as the vibration motion of the electron. However, they soon realize that they cannot explain the hydrogen spectrum observed earlier. Specifically, why it only has four frequencies and why the atom does not collapse (resulting in a continuous spectrum).

At this point, and after failing to find a solution to these problems, we tell the students that a satisfactory model cannot be found by using what classical physics establishes for the emission of electromagnetic waves. In this way, we make students participate in the crisis process that shocked physicists at the beginning of the 20th century, which is central for a correct understanding of the progress represented by quantum physics [53,54]. We ask the students to consider, as a hypothesis, the Bohr's postulates [55] and try again to explain the formation of the visible hydrogen spectrum:

- Electrons in atoms can only orbit in a few stationary states, characterized by fixed energies. In them, they orbit around the nucleus according to the laws of mechanics but without emitting energy.

- Any change in the atom involves an electron transition from one stationary orbit to another.
- The frequency of the emitted or absorbed radiation in a single transition depends on the initial and final energies, according to the expression $\left|E_{f}-E_{i}\right|=h \nu$, where $E_{i}$ and $E_{f}$ are the energies of the atom in the initial and final state, $v$ is the radiation frequency and $h$ is a constant.

Qualitative analysis leads them to consider the atom and the emission of radiation model proposed by Bohr to be adequate, but also to identify aspects that are contradictory to the classic model of electromagnetic waves emission. These contradictions force us to delve deeply into the explanatory capacity of the model before accepting it. In particular, we consider the following activities: (a) How can we use the model to interpret the fact that to ionize the hydrogen atoms, it will be necessary to illuminate them with light at a minimum frequency of $3.28 \times 10^{15} \mathrm{~Hz}$ ? (b) Can we deduce the frequencies of the hydrogen spectral lines? (c) Can we predict the existence of more nonvisible spectral lines? (d) How can we explain the hydrogen absorption spectrum? (e) How can we explain any other gas spectra (at least qualitatively)? (f) Energy quantization only occurs when atoms interact with radiation or is it an essential characteristic of the atom, regardless of how it interacts (Franck and Hertz experiment)?

The model established at this point includes key concepts $\mathrm{KC} 1$ and $\mathrm{KC} 3$, but the photon concept has not yet been introduced. We use the connection between the energy change in a single atom and the absorbed radiation frequency to highlight a contradiction: experimentally it is observed that each frequency of radiation can only produce a certain electronic transition, a connection that classical physics does not contemplate. This makes it reasonable, even if it contradicts the wave model of electromagnetic radiation, to think about the possibility that the energy in the radiation may also be quantified, i.e., to come in "packages" in which the amount of energy depends on the radiation frequency.

Testing this hypothesis (based on Einstein's in 1905) involves using it to try to solve problems that could not be solved with previous hypotheses and models. So, we start by presenting one of them: the photoelectric effect. We follow a similar strategy as previously: we familiarize the students with the phenomenon, highlight what could not be explained by classical theory, use Einstein's hypothesis to establish an interaction process between radiation and the electrons in the metal, design experiments, analyze data (obtained in experiments shown on video, if necessary), compare to the initial hypotheses in light of the results, and draw conclusions. This is how we came to establish the $\mathrm{KC} 2$ key concepts, which we strengthen by explaining the Compton effect, necessary to consolidate the corpuscular nature of photons (with linear momentum). Finally, we connect all the key concepts by reviewing and completing the previous explanation for the gas spectra. 
Although the established model satisfactorily explains emission and absorption of radiation, there are aspects that it cannot explain and that limit its validity. These limitations, which students should be aware of, are, among others, the existence of two contradictory models for electromagnetic radiation and the problem of predicting the existence of discrete energy states of atoms without resorting to ad hoc introductions from experimental data.

Before tackling these problems, we propose to the students activities in which they should use the quantum model of emission and absorption of radiation to explain phenomena such as fluorescence and phosphorescence or the operation of common technological devices such as neon lights, energy-saving lamps, LEDS, and lasers. These activities allow students to consolidate the established model, delve into the phenomena it can explain, and appreciate its predictive capacity.

After applying the established model, the TLS proposes activities for students to address one of the problems that limits its validity: the existence of two contradictory models for electromagnetic radiation. Specifically, the activities ask them to seek an interpretation for the phenomenon of radiation interference through a double slit to establish a single model that accounts for the behavior of photons. When they analyze what would happen if they carried out this experience with a single photon, the different groups of students realize that the place on the screen where this photon can be detected is indeterminate and subject to probabilistic interpretations. The final discussion in the class, guided by the teacher, concludes that the photon is generated as a corpuscle, is detected as a corpuscle, but in order to know where it can be detected it is necessary to analyze its movement as if it was a wave that indicates where it is more likely to be detected. This model can satisfactorily explain both radiation emission and absorption phenomena, as well as the interference phenomena. The TLS proposes activities to delve deeper into this same phenomenon, by analyzing how the distribution of radiation on the screen changes by modifying the characteristics of the double slit. The final discussion, guided by the teacher, comes to define the uncertainty principle.

Once the problem of the nature of radiation has been overcome, the TLS poses the problem of quantization in the atom: How to predict which orbits are stable for the electron? We ask the students to consider de Broglie's hypothesis, which states that the electron exhibits ondulatory behavior. The hypothesis implies that the stable orbits would be those in which the wave would be found as a standing wave. Testing the hypothesis requires demonstrating that the electron shows such wave behavior. The students, according to their previous experience with the wave model of light, suggest conducting a doubleslit interference experiment. After analyzing the results (photographs) in each group and after a discussion guided by the teacher, the students concluded that electrons (and by extension all particles) have a quantum wave behavior as described above for photons.

Finally, the TLS proposes activities to reflect on why we do not observe the particle's wave behavior regularly. Such activities provide opportunities for students to revisit previous experiments. After a group discussion, the students conclude that in all the experiments they have conducted before it has been necessary to make use of slits (or obstacles) of very small width, similar to the wavelength of the particles under study. Quantum phenomena will not manifest without such conditions, which are practically impossible in daily life. However, the dimensions of the atom are similar to the wavelength of the electron. From this conclusion, the teacher suggests that the wave function of the electron around the nucleus can be represented as a shaded area that tells us, depending on its "lightness" or "darkness," what is the probability of finding the electron. The teacher explains that this shaded area is called the "orbital."

The problem of studying in detail the atomic structure that arises from this electron model remains open. This aspect is no longer an objective of this course, according to the curriculum. Despite this, the TLS presents activities for students to reflect on the concept of orbital from the perspective of the "dual" or "corpuscle-wave" nature of the electron that defines quantum physics. This allows us, in our opinion, to connect and give a first physical foundation to the atomic model that the students used the previous year when studying chemistry and applying Moeller's rule.

The TLS was developed over 12 sessions, each lasting 55 minutes (3 weeks). Three sessions focused on atomic spectra and up to 8 on quantum phenomena of radiation emission and absorption. At the end of the TLS we expect the students to have acquired a quantum model of emission and absorption of radiation based on the Bohr model for the atom and on the photon concept for radiation. We also expect them to be able to identify which phenomena can be explained by using that model, under which conditions it was established and what its limitations are: to give response to other problems, such as the position or movement of an electron in the atom, this model is not sufficient and models based on the concept of the atomic orbital need to be used.

In Table II we present a summary of the TLS, relating how each problem contributes to build up the quantum model of emission and absorption of radiation and to address the aspects of scientific work for the students' methodological training.

\section{METHODOLOGY}

To assess the effectiveness of the TLS we compared the learning outcomes achieved by students treated with the 
TABLE II. Problem-based structure of TLS on the quantum model of emission and absorption of radiation.

\begin{tabular}{|c|c|c|c|}
\hline Problem sequence & $\begin{array}{l}\text { Procedures regarding science } \\
\text { to be learned by the students }\end{array}$ & $\begin{array}{l}\text { Explanations to be understood } \\
\text { by the students }\end{array}$ & $\begin{array}{l}\text { Activities } \\
\text { (see Ref. [56]) }\end{array}$ \\
\hline $\begin{array}{l}\text { Which phenomena produce } \\
\text { light? How can we change the } \\
\text { characteristics of the light } \\
\text { emitted in each of them? }\end{array}$ & $\begin{array}{l}\text { Become familiar with experimental } \\
\text { phenomena. Grasp the importance } \\
\text { of studying light emission and } \\
\text { absorption and its applications. } \\
\text { Need to plan a logical strategy for } \\
\text { moving forward. }\end{array}$ & $\begin{array}{l}\text { Highlight three key elements to } \\
\text { consider when searching for an } \\
\text { explanation: the substance or } \\
\text { material which emits or absorbs } \\
\text { radiation, the radiation (its spectrum } \\
\text { of frequencies and intensities), } \\
\text { and the interaction mechanism } \\
\text { between them. }\end{array}$ & A1 to A4 \\
\hline $\begin{array}{l}\text { Establishing a model that } \\
\text { explains the gas spectra. }\end{array}$ & $\begin{array}{l}\text { - Models must be tested in terms of } \\
\text { their ability to predict or explain. }\end{array}$ & Key concepts $\mathrm{KC} 1$ and $\mathrm{KC} 3$ & A5 to A14 \\
\hline $\begin{array}{l}\text { Is the energy in the radiation } \\
\text { quantified? }\end{array}$ & $\begin{array}{l}\text { - Before being accepted, a model must } \\
\text { prove its universality by explaining } \\
\text { and predicting phenomena that differ } \\
\text { from any used to establish it. }\end{array}$ & Key concepts KC2 & A15 to A23 \\
\hline $\begin{array}{l}\text { Review of progress-Limits on } \\
\text { the validity of the established } \\
\text { model }\end{array}$ & $\begin{array}{l}\text { The scientific models have a limit of } \\
\text { validity. They have been set after } \\
\text { accepting idealizations and } \\
\text { simplifications. They cannot be used } \\
\text { when the conditions under which } \\
\text { they were set are not met. }\end{array}$ & $\begin{array}{l}\text { - We cannot predict the energy values } \\
\text { for stationary atomic states. The } \\
\text { model is an ad hoc construction. } \\
\text { - We cannot explain wave phenomena } \\
\text { such as interference or light } \\
\text { diffraction. }\end{array}$ & Recapitulation \\
\hline $\begin{array}{l}\text { Possible applications of the } \\
\text { developed model }\end{array}$ & $\begin{array}{l}\text { Scientific models are representations } \\
\text { that allow us to simplify complex } \\
\text { phenomena, reason, visualize } \\
\text { abstract entities and interpret and } \\
\text { predict results. }\end{array}$ & $\begin{array}{l}\text { Emission and absorption phenomena } \\
\text { can be explained using the same } \\
\text { model: this requires analyzing the } \\
\text { structure of energy states of the } \\
\text { atoms, molecules or crystals } \\
\text { involved, determining the energy } \\
\text { and quantity of photons that make up } \\
\text { the radiation and establishing an } \\
\text { interaction mechanism between } \\
\text { them. }\end{array}$ & A24 to A28 \\
\hline $\begin{array}{l}\text { Light is ondulatory or } \\
\text { corpuscular? }\end{array}$ & $\begin{array}{l}\text { - Several models may be necessary to } \\
\text { represent entities and phenomena } \\
\text { (i.e., atoms). } \\
\text { - Each model responds to a well- } \\
\text { defined set of problems. }\end{array}$ & $\begin{array}{l}\text { Photons are emitted and absorbed as } \\
\text { corpuscles. Predicting at what point } \\
\text { they will be detected requires } \\
\text { understanding their propagation as } \\
\text { that of a wave. }\end{array}$ & A29 to A31 \\
\hline How to predict stable orbits? & $\begin{array}{l}\text { - Experts use different models, } \\
\text { identifying the problems to which } \\
\text { they give response and their limits of } \\
\text { validity. }\end{array}$ & $\begin{array}{l}\text { Electrons are detected as corpuscles. } \\
\text { Predicting at what point they will be } \\
\text { detected requires understanding their } \\
\text { propagation as that of a wave. }\end{array}$ & A32 to A35 \\
\hline
\end{tabular}

TLS (experimental group) and students who learned quantum physics through habitual teaching (control group):

- The TLS was implemented with 5 cohorts of physics students from the last year of high school, featuring a total of 74 students, over 4 years, in two high schools in the province of Alicante (Spain). One of them is a city-center school located in Alicante, the provincial capital city with 330000 inhabitants, while the other is a city-center school in a city of 100000 inhabitants located less than $100 \mathrm{~km}$ from Alicante. Four out of the five groups were taught by the lead author of this paper and the other was taught by a collaborating teacher who has expert knowledge of the TLS. The collaborating teacher used the program of classroom activities with the students [57] and the teacher's guide $[56,58]$. This teacher's guide includes justification of the sequence of activities, the goal of each activity, expected answers for each activity, and the answers given by the students in the first year of implementation. The collaborating teacher had the opportunity to discuss all aspects of the TLS with the lead author of this paper, before and during implementation. Both teachers have over 10 years of experience as high school physics teachers and for most of this time they have been teaching using problem-based teaching and learning strategies. 
- The control group is made up of 67 students, from 4 cohorts, who studied the unit following the usual teaching methodology: they follow a textbook (similar to the textbooks for college [59]) that contemplates in a unit the contents and objectives established by the official curriculum. Of the 4 weekly class sessions, 2 hours are devoted to lectures and 2 hours to recitation (problem solving). No time is devoted to experimental work. In the course, students do not normally have the opportunity to participate actively and limit themselves to taking notes from the teacher's explanations, both in lectures and in problem sessions. The students' role was limited to asking questions for clarification after teacher explanations.

The 67 students receiving conventional teaching (control) and the 74 students who followed the TLS attend similar schools and devote the same amount of instructional time to the quantum phenomena of emission and absorption of radiation.

To evaluate the learning achievement reached by the experimental and control students, a pretest and post-test were designed with very similar open questions. The posttest questions, that have already been validated in another study [19], are attached as the Appendix. It includes three questions:

- The first question Q1 (see the Appendix) looks at understanding the absorption of radiation phenomenon. $\mathrm{KC} 1, \mathrm{KC} 2$, and $\mathrm{KC} 3$ are assessed in it. A correct answer should state that atoms can only be found in stationary states (KC1) and that the transitions between them are caused by the absorption of indivisible photons (KC2). In addition, the energy of a single photon cannot be absorbed by more than one atom, nor can an atom absorb several photons in a single transition. Following this line of reasoning, an atom in the fundamental state will make a transition to a higher energy state if it absorbs a photon with energy that is equal to the difference between the initial and final states (KC3). For question Q1, photons of $10.2 \mathrm{eV}$ will be absorbed, producing a transition from the $-13.6 \mathrm{eV}$ state to the $-3.4 \mathrm{eV}$ state. Photons with $13 \mathrm{eV}$ may not be absorbed because they would move the atom to an energy state that does not correspond to any stationary state.

- In question Q2 (see the Appendix), we analyze whether students can predict the emission spectrum detected after hydrogen atoms are excited to a welldefined state. The correct answers should take into account that atoms in an excited state can only make transitions to lower energy states (KC1). These transitions are random, i.e., they can occur to any lower energy state. When a single hydrogen atom makes a transition, it emits a single photon (KC3). For the case stated in the question, transitions can take place from -1.51 to $-3.4 \mathrm{eV}$ or from -1.51 to
$-13.6 \mathrm{eV}$. A single photon is emitted in each one. Atoms that have moved to the $-3.4 \mathrm{eV}$ state will later make a transition to the $-13.6 \mathrm{eV}$ state, emitting a second photon. In the emission spectrum, photons with three energies, and therefore three frequencies, will be detected. Three lines will appear, with an intensity that depends on the probability of each transition.

- Question Q3 (see the Appendix) focuses on the interpretation of spectral lines intensity and explores key concepts $\mathrm{KC} 2$ and $\mathrm{KC} 3$. A correct answer to the question should involve matching a more intense line to a higher energy per unit of time of the amount of photons that have the frequency of the spectral line $(\mathrm{KC} 2)$. As each photon has been generated in a random transition, a line of higher intensity also indicates that the transition which generates these photons is more likely to occur than the others (KC3). In the case considered, the most intense line is the yellow one and its frequency corresponds to the lowest photon energy, so the number of photons with that frequency emitted in a second should be higher than the others. This is equivalent to considering the atomic transition that gives rise to the photons with the yellow line frequency to be more probable than the others. A similar analysis will explain the other spectral line intensities.

Although the students had not previously studied a quantum model of emission and absorption of radiation, we carried out a pretest to assess their initial knowledge before starting the TLS. The phenomena being presented and the conceptual questions were the same in the pretest and post-test, so that the objectives of each question can be considered equal, but in the post-test, numerical aspects were included that it did not make sense to include in the pretest. The pretest was validated by four teachers (three high school teachers and one university teacher). All the high school teachers stated that they expected that no student would give a single correct answer before studying the quantum physics unit. The questionnaire was handed out by the lead author of this paper to all the groups, in the presence of the group's teacher and during a session prior to starting the study of the quantum physics unit. The students answered the questionnaire under examination conditions (without the possibility of consulting peers, textbooks, or other materials) and did not experience any difficulty in understanding the statements, although they did not know what to answer. They spent about 10 minutes on the questionnaire.

One month after finishing the TLS, we gave a questionnaire to the students as a post-test. The questionnaire was handed out during a session by the lead author of this paper. The students answered with interest, under test conditions, and spent about 20 minutes on it. In general, they did not experience any difficulties in understanding the text or the content of the questions. 
Two days after the questionnaires were completed, we interviewed 5 students from the experimental groups who had obtained particularly good results in the unit's evaluation exams and who had answered the post-test questionnaire correctly. For the interviews among the experimental students we used the answers they gave to the questionnaires. At the beginning of each interview, students were shown the answers they had given in the questionnaires and were asked questions such as "What do you mean by this answer?", "How do you imagine the atoms that make up the gas and what happens to them when they interact with radiation?", "Could you explain this phenomenon considering that atoms are made up of particles like protons, neutrons, and electrons?". These interviews intended to analyze in greater depth the understanding of the quantum model of emission and absorption of radiation acquired by the students. It also pretends to assess whether they had memorized how to use it or, on the contrary, they have understood the phenomena and their own explanations about it. The interviews lasted about 10 minutes and were conducted by the lead author of this paper.

The answers obtained in the pretest and post-test questionnaires were initially analyzed by the first author of this paper. The kappa intrarater reliability coefficient was calculated by this researcher three weeks later, obtaining a value of 0.86 on average for all questions, satisfactory for a level of confidence of $95 \%$. Then, the lead researcher proposed a draft set of categories for each of the questions. Subsequently, all researches read the answers and each response was tentatively assigned a category. Then, the results were discussed with the other members of the research team. The interrater kappa Cohen reliability coefficient averaged 0.84 for the questions, indicating a very significant degree of agreement in the judges' criteria for assigning the categories used to interpret the responses. When there was disagreement about a description category or the location of responses to a specific category, this was resolved by just using evidence of students' understanding as a reference [60].

The answers to the questions were grouped into the following categories:

A. Correct answer and explanation to the question.

B. The answer includes one or more of the difficulties: B1. Difficulties related to $\mathrm{KC} 1$

B2. Difficulties related to $\mathrm{KC} 2$

B3. Difficulties related to KC3

And/or a new difficulty.

C. Unclassifiable. Explanations without logical consistency.

D. No answer.

The classification was necessary to analyze the impact of the TLS in a qualitative way, as we do in the next section. However, to conduct a quantitative comparison of the percentage of correct and incorrect answers in each group, we have calculated the $\chi^{2}$ value in each question and the associated $p$ value. To do so, a contingency table was obtained by collapsing the categories B, C, and D into one unique "incorrect answer" category to meet chi-square assumptions. Moreover, we have also calculated the $p$ value using Fisher's exact test and we have analyzed the odds ratio and the Cohen's $h$ value.

The interviews were transcribed and everything that the students wrote and drew was kept for subsequent analysis. The interviews were analyzed independently by three authors of this paper. Passages were classified in two initial groups, based on the degree of understanding regarding the model of emission and absorption of radiation. By doing this initial separation, some doubts in the evaluation process were solved and, moreover, some examples of how students use the model could be assessed [61].

\section{RESULTS}

\section{A. Results from the questionnaires}

In the pretest, no student, either from the control group or from the experimental one, answered any of the questions correctly. Only a small percentage of students in both groups gave answers that match classical interpretations of spectra. In addition, a few students used terms from quantum physics incorrectly, showing that they remembered memorized knowledge on atomic theory studied in chemistry. The vast majority of students in both groups did not answer the questions or their answers were not logical. In accordance with the results obtained, we can state that the students in both groups had no knowledge of quantum physics. So, in terms of previous knowledge of the topic, the control and experimental samples are uniform.

In Table III we present the percentage of students' correct responses in the experimental and control group to post-test questions, as well as the values obtained for the statistics used. The statistical parameters used are suited to compare the effect of an innovation in the experimental and control groups [62]. We have calculated the $\chi^{2}$ value in each question, and the associated $p$ value, by using a contingency table with the number of students that answered correctly and incorrectly. Although the sample size is sufficient to apply $\chi^{2}$, we have also calculated the $p$ value using Fisher's exact test. Finally, we calculated the odds ratio and the size of the effect using Cohen's $h$. No statistical differences have been observed between the students in the 5 experimental cohorts so they have been grouped together into a single experimental group $(N=74)$. The same happens with the students from the control cohorts and the results have also been grouped together into a single control group $(N=67)$. Figure 1 shows the percentage of correct answers of the experimental and control group to each question.

In the question referring to absorption (Q1) the applied statistics show that there is a statistically significant 
TABLE III. Percentage of correct answers to each question by the experimental groups (E group) and control groups (C group). Statistical comparison between them.

\begin{tabular}{lcccccr}
\hline \hline Question & $\begin{array}{c}\text { Percentage of correct } \\
\text { answers (E group) }\end{array}$ & $\begin{array}{c}\text { Percentage of correct } \\
\text { answers (C group) }\end{array}$ & $\begin{array}{c}X^{2}\left(\chi^{2}{ }_{0.95}=3.84 ;\right. \\
\mathrm{df}=1)\end{array}$ & $P$ (from $\left.\chi^{2}\right)$ & $\begin{array}{c}P \text { (from } \\
\text { Fisher test) }\end{array}$ & Cohen's $h$ \\
\hline Q1 & 66.2 & 9.0 & 48.46 & $<10^{-5}$ & $<10^{-5}$ & 1.29 \\
Q2 & 59.5 & 7.5 & 41.93 & $<10^{-5}$ & $<10^{-5}$ & 1.21 \\
Q3 & 64.9 & 9.0 & 46.51 & $<10^{-5}$ & $<10^{-5}$ & 1.26 \\
\hline \hline
\end{tabular}

difference between the results obtained by the experimental group and the control one. By comparing the percentage of correct and incorrect answers in each group we obtain a $\chi^{2}=48.46$, higher than the critical value for 1 degree of freedom $\chi_{0.95}^{2}=3.84$, and $p<10^{-5}$. The result is consistent with that obtained by applying the Fisher's test, which also provides a $p<10^{-5}$. We obtain an odds ratio of 19.93, which means a large gain of the experimental group compared with the control group. Also, the value of Cohen's $h=1.29$ indicates a large effect size. In question Q2, referring to emission, the differences between the results of the experimental and control group are significant when applying the statistics. By comparing the percentage of correct and incorrect answers for each group, we obtain $\chi^{2}=41.93$ and $p<10^{-5}$ (critical value for 1 degree of freedom $\chi_{0.95}^{2}=3.84$ ). Fisher's test also indicates that $p<10^{-5}$. The calculated odds ratio value is 18.19 . The size of the effect achieved in this aspect when applying the TLS is large, as shown by the Cohen's $h$ value, $h=1.21$. In Q3 (referred to the intensity of the spectral lines), we observe that the differences are also statistically significant when comparing the percentage of correct and incorrect answers given by each group using the three statistics. We obtain a $\chi^{2}=46.51$ higher than the critical value (critical value for 1 degree of freedom, $\chi_{0.95}^{2}=3.84$ ), corresponding to $p<10^{-5}$. Fisher's test also indicates that $p<10^{-5}$. The odds ratio value is 18.77 . Cohen's $h$ has a value of $h=1.26$, indicating a large effect size [63].

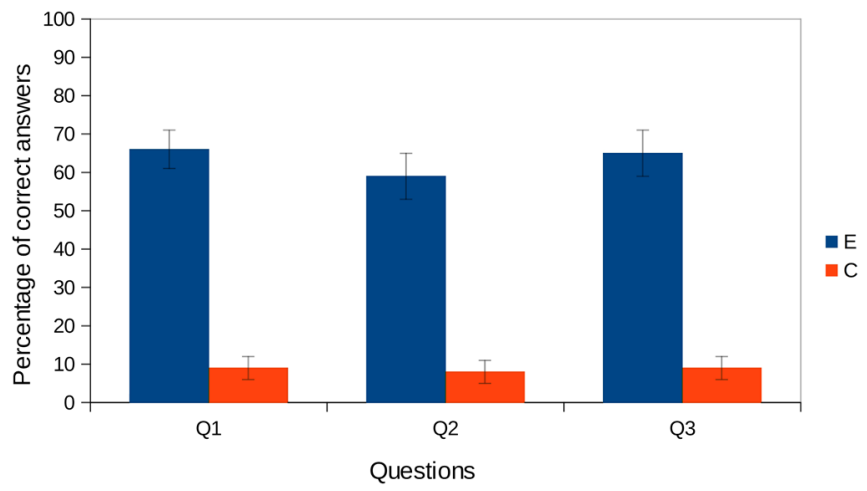

FIG. 1. Percentage of correct answers given to each of the questions by the 74 experimental students (E) and the 67 control students (C).
After analyzing the percentage of correct answers to each question separately, we carried out a global analysis. In order to do this, we have assigned the students one point for each question that they have answered correctly. In Table IV we show the percentage of students who respond correctly to $0,1,2$, or 3 questions (getting $0,1,2$, or 3 points, respectively) for the experimental and control groups. $41 \%$ of the experimental students answer the three questions correctly, while no control student manages this. In addition, we should highlight that the mode for the experimental students corresponds to have three correct answers, while it corresponds to 0 correct answers in the case of the control students.

We have conducted a Shapiro-Wilk test to assess to what extent the quantity of correct answers for the experimental and control group match a normal distribution. We have obtained $W_{\text {Exp }}=0.815$ and $W_{\text {Cont }}=0.54$ for the experimental and the control group, respectively. Both values correspond to a $p$ value lower than $10^{-5}$ and are not included into the interval of confidence of $95 \%$ of probability $(0.9670,1)$, so we have to conclude that the distributions do not match a normal distribution. Taking that result into account, we have conducted a MannWhitney nonparametric test to compare the results. The value obtained for this statistic was $U=661.5$. It corresponds to a $p$ value lower than $10^{-5}$. It is equivalent to a $Z=7.5$ in a normal distribution, higher than the critical value of 1.9599 for two tails. Although the occurrence of only 3 correct answers may weaken the value of the statistic, we consider that the statistically significant differences are consistent with the results presented above for each individual question and show the global impact of the TLS.

TABLE IV. Percentage of students from each group categorized by the quantity of correct answers.

\begin{tabular}{lcc}
\hline \hline $\begin{array}{l}\text { Quantity of correct } \\
\text { answers (punctuation) }\end{array}$ & $\begin{array}{c}\text { Experimental } \\
\text { percentage of } \\
\text { students }\end{array}$ & $\begin{array}{c}\text { Control } \\
\text { percentage of } \\
\text { students }\end{array}$ \\
\hline 0 & 17.6 & 77.6 \\
1 & 17.6 & 19.4 \\
2 & 24.3 & 3.0 \\
3 & 40.5 & 0.0 \\
Mean (SD) & $1.88(1.13)$ & $0.25(0.50)$ \\
\hline \hline
\end{tabular}


TABLE V. Percentages of learning difficulties in experimental students (E) and control students (C).

\begin{tabular}{lcccccc}
\hline \hline & $\begin{array}{c}\text { Q1: } \\
\text { absorption }\end{array}$ & $\begin{array}{c}\text { Q2: } \\
\text { emission }\end{array}$ & $\begin{array}{c}\text { Q3: } \\
\text { intensity }\end{array}$ \\
\cline { 2 - 7 } & $\begin{array}{c}\text { (\% of } \\
\text { answers) }\end{array}$ & $\begin{array}{c}\text { (\% of } \\
\text { answers) }\end{array}$ & $\begin{array}{c}\text { (\% of } \\
\text { answers) }\end{array}$ \\
\hline Category & E & C & E & C & E & C \\
\hline B1. Difficulties with KC1 & 8.1 & 9.0 & 1.3 & 1.5 & $\ldots$ & $\ldots$ \\
B2. Difficulties with KC2 & 0.0 & 3.0 & $\ldots$ & $\ldots$ & 4.0 & 23.9 \\
B3. Difficulties with KC3 & 12.2 & 31.3 & 12.2 & 20.9 & 14.9 & 13.4 \\
C. Unclassifiable & 10.8 & 16.4 & 25.7 & 28.3 & 14.9 & 23.9 \\
D. Did not answer & 2.7 & 31.3 & 1.3 & 41.8 & 1.3 & 29.8 \\
\hline \hline
\end{tabular}

Regarding students' difficulties in learning the key concepts of the TLS (see Table I), we are interested in looking at how learning difficulties have changed in relation to the key concepts. We have classified the incorrect answers (Table V). Category B comprises the ties in relation to the key concepts. We also found some incorrect answers not clearly related to any key concept. In many cases these lacked internal logic. None of them were detected with a percentage higher than $3 \%$, so we have classified them in category $\mathrm{C}$ as "unclassifiable." Finally, category D corresponds to answers left blank.

The difficulty B1 (related to KC1) was found mainly in questions Q1. It includes answers that explicitly show not understanding that the energy is quantized in the atoms (KC1). Students who gave those answers mostly consider that the atom can absorb any photon and reach an energy different from that of the stationary states. The percentage of students for difficulty B1 is about $10 \%$ in both groups. There does not seem to be any progression between experimental and control regarding this difficulty.

The difficulty B2 was mainly found in question Q3. It corresponds to answers where the intensity of the radiation is not related to the quantity of photons within it, always attributing higher intensity to the radiation formed by photons with higher energy.

Regarding the difficulty B3, the students use alternative explanations depending on the context of the question (Q1, Q2, and Q3) but in all cases, the answers show that students did not understand $\mathrm{KC} 3$.

- In all the questions, a set of explanations are based on the fact that the energy diagram represents both the energy of the stationary states and the energy of the photons that can be absorbed (as an absolute value). So, only photons that match the energy values shown in the diagram are emitted or absorbed.

- In question Q1, a set of explanations ignores the fact that the energy conservation principle must be accomplished in the interaction processes between photons and electrons: students that answer in this way indicate that the atom, when it absorbs the photon, performs a transition that takes it to a stationary state, whose energy difference from the initial state does not coincide with the photon's energy. Moreover, they do not give any explanation of what happens with the "missing" or "exceeding" energy.

- In question Q2, a set of explanations omit that transitions between stationary states are random and it indicates that the spectrum will have a single line, corresponding to the light emitted by the atoms when making the transition from the excited state directly to the fundamental.

- In question Q3, a set of explanations consider that the most intense radiations are those emitted by the atoms found in the states of higher energy (in absolute value), without considering that the atom has to make a transition when it emits radiation.

Fluctuations in the percentages are not considered in absolute values, no statistical differences are sought, but an attempt is made to observe changes in the difficulty distribution shown in Table V. The percentage of answers left blank by control students is particularly high compared to an insignificant percentage in the experimental group. The lack of answers, also detected by other previous studies $[15,19]$, can be attributed to the lack of an explanatory model that helps them find and/or justify their answers. Regarding the difficulties related to the key ideas, we observe that the percentage of difficulties detected in the experimental students is lower in all cases, except for difficulty B3 for question Q3 that remains constant. The differences related to difficulty B3 in question Q1 and the differences related to difficulty B2 in question Q3 are particularly noteworthy. This analysis gives us clues about the weakest parts of the TLS, pointing to possible improvements in both its design and its implementation.

\section{B. Interviews with the experimental students}

After reviewing the answers given in the questionnaire by the experimental students, we interviewed five of them to assess the quality of the students' argumentation when explaining their answers and the scientific model they use. Consequently, five students who had answered the three questions correctly where selected, to assess to what extent they really understood their given answers or, in contrast, if they had simply given algorithmic answers, without understanding the processes involved.

The answers given by the students in the interviews show that they can use various representations of the atom coherently (establishing relationships between them) to explain what happens when radiation interacts with atoms. Student 7 (we used numbers to preserve anonymity) answers as follows when asked to use another representation of the hydrogen atom to Q1 [Fig. 2 shows the answer (s)he gave in the questionnaire and the picture (s)he draws in the interview]: 


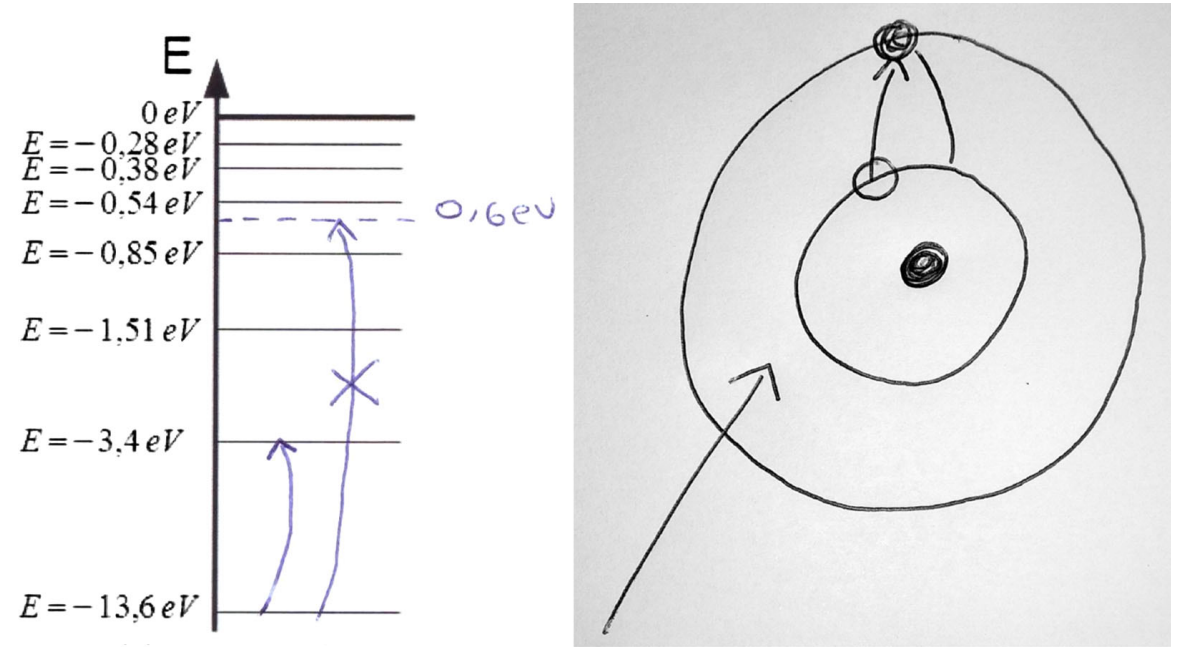

FIG. 2. Student 7's answer to Q1 (left) and representation of the atom made during the interview (right). (S)he draws a nucleus and an electron that makes a transition to a more external orbit when it absorbs a photon (represented by an arrow coming from below). (S)he uses a line to represent the transition that will result in emission of radiation. Student 7's written response: "Subparagraph a) The atoms absorb the photons from light and they will jump to the stationary state of $-3.4 \mathrm{eV}$, then they will go down again emitting light. Subparagraph b) There will be no jump, it is impossible because there is no stationary state of the hydrogen atom at $-0.6 \mathrm{eV}$, so the photon will pass by.

"Interviewer: What image do you have of atoms? What do you imagine, a lot of energy diagrams inside the vessel?

Student 7: No, I suppose that a single diagram. A single diagram for a given gas.

I: Then, regarding those atoms in there, considering that they are formed by protons, neutrons, and electrons, would you be able to draw any of those atoms?

St7: I suppose it would be an atom containing its corresponding protons and electrons. But together $[(s)$ he draws a point and an orbit around it representing an electron that orbits a nucleus, as happens with hydrogen]. And when emitting light, it would propagate accordingly.

I: So, when the atom absorbs light, what would happen to the atom?

St7: Well, by absorbing light, if the light is sufficient for the electron to go into a stationary state, then I suppose the electron will absorb it and later, it will shift to a state of lower energy and emit light [he draws an arrow that refers to the radiation incident on the atom and, by means of another arrow, the transition of the electron into a more external orbit, then (s)he adds a line that represents the transition back into the internal orbit, which would correspond to the aforementioned emission].

I: If the atom you drew was the atom in the base state, what would it be like after absorbing radiation?

St7: It will shift to a... to another stationary state if the light is enough for it to do so. Then it will shift again to any other lower energy state.

\section{I: What if the light weren't absorbed?}

St7: Nothing would happen. I suppose the light would pass by and have no effect on the atom."

Two of the students interviewed go one step further and refer to the quantum model of the atom instead of using Bohr's model, using the concept of orbital instead of orbits. As an example, we are showing the answers given by Student 22. Here we can observe that (s)he uses different representations to refer to the atom according to the situation being approached and the feature (s)he intends to emphasize:

"Interviewer: The atoms in there, the hydrogen atoms inside the vessel, how do you imagine them?

Student22: Dispersed, scattered over there. No?

I: Lots of scattered atoms? Could you draw those atoms?

St22: I don't know, like it's all filled up, like this, a lot [(s)he represents small dots inside a vessel, a representation that looks like the kinetic-corpuscular model of a gas].

I: And when those atoms receive radiation, what do you think happens to them?

St22: Well, then that happens [(s)he points out his answer in the questionnaire, the energy diagram], that the electron changes from... well, it makes a transition, doesn't it?

I: That's the answer you gave. But I would like you to think of atoms made up of protons, neutrons and electrons, or do you imagine that there are energy diagrams in the vessel? 
St22: No, no, no.

I: Protons, neutrons and electrons, how do you imagine them in the atom? What do you think that happens to the atom?

St22: I know they're not going to be moving in circles, but it's more like they're in one of those orbitals....

I: Could you represent the atom in any way?

St22: Let's see, we represent it like this [(s)he draws an electron making a circular movement around a proton] but really, apart from the fact that there is no [orbit as the electron's path] the electron is where there is more... you see, the orbital is where the electron is most likely to be, isn't it? There are different ones.

I: So, there are no orbits, there are orbitals.

St22: Yes.

I: And using this atom model, when that atom absorbs radiation, what happens to it?

St22: That... It sort of gets bigger [(s)he draws the electron further from the nucleus]. Because of the attraction, it goes down again and emits radiation."

We observe that the student combines up to 4 representations for the atoms contained in the vessel according to the feature he intends to describe: (s)he imagines the set of atoms as dispersed points inside the vessel (according to the kinetic-corpuscular model), (s)he uses the energy diagram to reason about the transitions taking place in the atom, (s)he refers to electrons orbiting the nucleus to interpret the change in the structure of the atom after absorbing radiation, but (s)he refers to the atomic orbitals when referring to the position and absence of trajectory of the electron. We observe, moreover, that the representations are not mixed in an unreflective way, but that each one responds to a different problematic aspect.

We get similar explanations by asking students for more details about question Q2, as shown in the following example. In Fig. 3, we show the drawings made by the student:

"Interviewer: In this second question about emission, if we take into account that atoms are made up of protons and electrons, as you have represented before, can you explain what would have to happen to the atom for radiation to be emitted? In this case, what's happening to the atoms?

Student 20: They're returning to their minimum energy state.

I: Could you represent this in some way with drawings? St20: Let's see, if... if this is where it is once the electric discharge takes place $[(s)$ he draws an atom with a proton and an electron and, with an arrow, represents the transition to a more external orbit] well, it would return to its state... I don't think I will do it the same as the previous one [in reference to the size of the drawings]. I: Yes, we admit that the drawings are approximate. St20: Yes [(s)he uses an arrow to represent transition from the outermost to the innermost represented orbits].

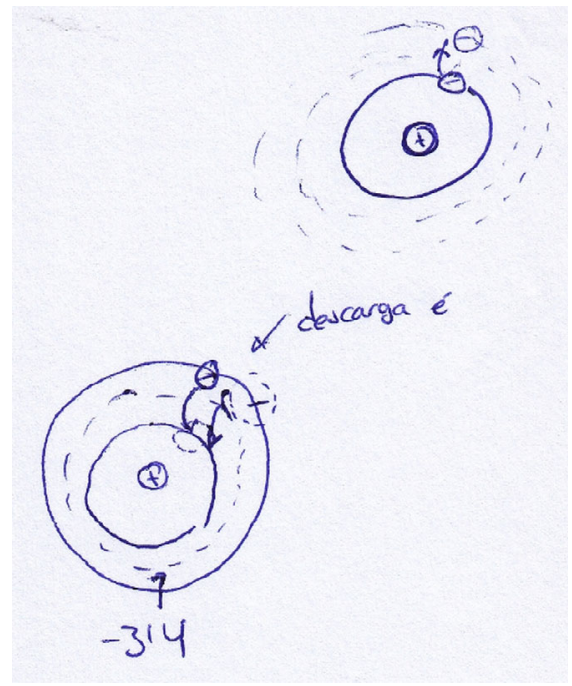

FIG. 3. Drawing by student 20 to explain atomic transitions and how they relate to the emission spectrum. At the top, (s)he shows the absorption of energy produced by the electrical discharge and at the bottom, (s)he shows the possible transitions back to the fundamental state.

I: Would it return to its fundamental state? St20: Exactly.

I: But here you tell me that there are three transitions [in reference to his written response to the question].

St20: Three possible, that is, it could go either directly or through the $-3.4 \mathrm{eV}$ state, let's pretend there is another electron, we know there isn't but... [(S)he adds an intermediate orbit between the previous two and an electron that makes a transition from it to the innermost orbit. [(S)he says he does so to avoid representing another atom but ( $s$ )he recognizes that hydrogen only has one electron].

I: Would it be another atom?

St20: Oh, well, yes. Exactly. Firstly, It'd do the.... The shift to the middle state, so to speak, and then It'd go back to the base state."

We can see that the student correctly relates the energy diagram to the pictorial representation of the atom and that (s)he is able to pass from one to the other according to the feature (s)he wants to emphasize [(s)he uses the energy diagram to work with quantitative values and the representations based on the Bohr model to qualitatively explain what happens to the atoms].

\section{CONCLUSIONS}

In this research, we set out to design and evaluate a problem-based TLS to improve teaching and learning of quantum physics in introductory courses, specifically focusing on atomic spectra. We have designed the TLS considering difficulties that students are unable to overcome when studying atomic spectra in regular education. 
In addition, we have developed a teacher's guide [56,58] that explains the goals of each activity, the main students' difficulties when carrying them out, and the strategies to overcome these difficulties.

Results from the administered questionnaire show that there is a significant improvement in the students' learning, which has been analyzed in light of the key ideas of the quantum model of emission and absorption of radiation. This improvement is characterized by a significant increase in the students' ability to use the quantum model of emission and absorption of radiation to interpret frequencies and intensities of radiation in emission and absorption phenomena. By interviewing some experimental students we have shown that they understand the quantum model of emission and absorption of radiation and they are able to select the representation of the atom they use taking into account the aspects they want to analyze or emphasize. They use energy diagrams to deal with the quantitative aspects. They use pictorial representations to explain what happens to subatomic particles during emission and absorption. Likewise, they resort to the Bohr model due to its simplicity but recognize that the quantum model of the atom, with atomic orbitals, must be used to interpret magnitudes such as the position of the electron in the atom.

Results also show the necessity for some changes in the TLS. Three are the main points we are working on to improve its efficacy:

- Reducing the percentage of incorrect answers corresponding to $\mathrm{KC} 3$. It seems that a further insight on the emission and absorption of radiation processes is required in order to overcome those difficulties. We should require the students to use the energy diagram to represent and discuss each phenomenon avoiding direct interpretations using unique equations or quantitative operations.

- Increasing the emission phenomena interpretation capability. Q2 referring to the emission spectrum registered the lower correct answers' percentage and the higher nonclassifiable amount of response. Matching electrons' transitions in the atom and spectral lines is hard for the students, as other studies have shown [23]. An effort should be made to introduce activities aiming at reinforcing that point.

- Improving the use of the orbital atomic model. Almost all devices and applications of the emission and absorption of light have been studied after introducing the quantum model of emission and absorption of light and before introducing the orbital model of the atom. We consider that some "application activities" could be moved to the end of the unit, bringing the students opportunities to use the recently introduced atomic model and to overcome the use of quasiclassical models.

We are aware that the student samples in this study are not extensive, but some of the students' difficulties that we detected concerning emission spectra match to other international previous studies $[15-17,20]$. In addition to them, we have also focused on absorption phenomena and on emitted light intensity. Because of the difficulty of finding control groups that perform classroom analysis of the quantum phenomena of emission and absorption of radiation other than gas spectra and the photoelectric effect, we have limited the learning evaluation questionnaire to gas spectra. We are currently working on extending this research to assess the impact of the unit more extensively, including the students' capability to explain more quantum phenomena and expanding student samples to university degrees.

As we mentioned in the introduction, the use of the Bohr model in teaching quantum physics is controversial [25-27,64-68]. This and other quasiclassical models have been identified to be the origin of difficulties and alternative ideas held by the students in advanced quantum physics courses and that are difficult to overcome [25-27]. The importance of the correct learning of Bohr's model on subsequent learning of quantum concepts has also been noted [68]. Despite all this controversy, we must bear in mind that using atomic models based on Schrodinger's equation can be excessively complicated for introductory quantum physics courses in high school. By following the TLS, the students have learned to use Bohr's model to interpret radiation emission and absorption phenomena. They have done so by facing a path of problems that allows them to recognize the experimental situations to which it responds, the simplifications on which it has been established, and the problems that it cannot explain and that limit its use. This helps students to choose it as suitable for explaining certain phenomena, but they resort to other more suitable models or representations when this model fails. This ability corresponds to expert thinking [5,10-14].

As we stated in the introduction to this paper, scarce research has been done in physics education on quantum phenomena of emission and absorption of radiation, and the little research in this field focuses on gas spectra and on the photoelectric effect [18]. Concerning introductory quantum physics, computer-assisted activities have been reported to have brought positive results on students' learning $[3,4,14]$. However, these studies also report persistent student difficulties when working with models $[4,14]$. Focused only on emission spectra, the researchbased tutorial of Ivanjek et al. [23] brought positive results on students learning. Our research provides a new proposal to address this subject that considers a greater diversity of emission and absorption of radiation phenomena and that has, as we have shown, a positive impact on learning about models and atomic spectra. The similarities and differences between our results and previous research lead us to think that our experience may be useful in educational contexts for introductory quantum physics courses, in both high school and college. 


\section{APPENDIX: POST-TEST QUESTIONNAIRE}

In Fig. 4 we present the questions and figures used in the post-test.

Q1- (Absorption spectra) The diagram on the right shows the energy of the hydrogen atom stationary states.

There are millions of hydrogen atoms in a vessel, all of which are in the lowest energy state, i.e. in the base state.

a) If we irradiate the vessel with photons that have $10.2 \mathrm{eV}$, what will happen to the gas atoms?

b) If we irradiate the vessel with photons that have $13 \mathrm{eV}$, what will happen to the gas atoms?

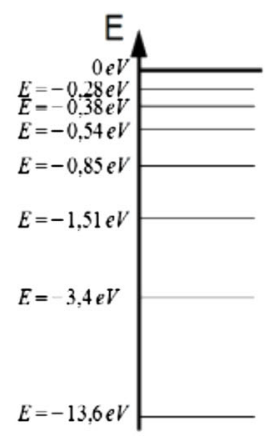

Q2- (Emission spectra) Hydrogen atoms contained in a vessel are excited by an electric discharge to the $-1.51 \mathrm{eV}$ stationary state (the diagram on the right shows the energy of the hydrogen atom stationary states). A spectroscope is then used to analyze the light emitted by the gas. What will be observed? (Choose one):

a) A single-colour line (light with a single frequency).

b) Multi-colour lines (light with a few frequencies).

c) A continuous spectrum, like a rainbow (light with all frequencies).

Back up your answer.

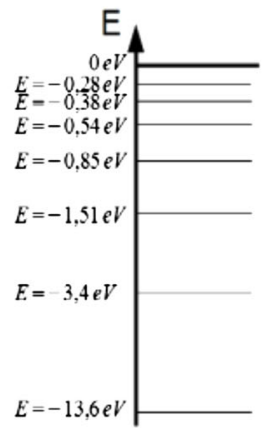

Q3- (Spectra line intensity) How can you explain that there are some lines with higher intensity than others in a gas spectrum, as you can see in the picture of the Helium spectrum obtained in a high school laboratory?

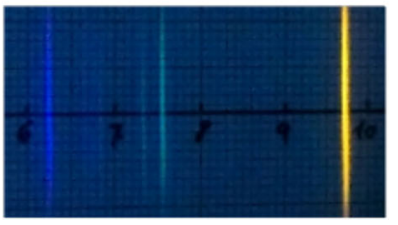

FIG. 4. Questions and figures presented in the post-test.

[1] J. Petri and H. Niedderer, A learning pathway in highschool level quantum atomic physics, Int. J. Sci. Educ. 20, 1075 (1998).

[2] V. Olsen, Introducing quantum mechanics in the upper secondary school: a study in Norway, Int. J. Sci. Educ. 24, 565 (2002).

[3] D. A. Zollman, N. S. Rebellos, and K. Hogg, Quantum mechanics for everyone: Hands-on activities integrated with technology, Am. J. Phys. 70, 252 (2002).

[4] S. B. Mc Kagan, K. K. Perkins, M. Dubson, C. Malley, S. Reid, R. Le Master, and C. E. Wieman, Developing and researching PhET simulations for teaching quantum mechanics, Am. J. Phys. 76, 406 (2008).

[5] S. B. Mc Kagan, K. K. Perkins, and C. E. Wieman, Why we should teach the Bohr model and how to teach it effectively, Phys. Rev. ST Phys. Educ. Res. 4, 010103 (2008).

[6] H. K. E. Stadermann, E. van den Berg, and M. J. Goedhart, Analysis of secondary school quantum physics curricula of 15 different countries: Different perspectives on a challenging topic, Phys. Rev. Phys. Educ. Res. 15, 010130 (2019).

[7] H. Kragh, Quantum Generations. A History of Physics in the Twentieth Century (Princeton University Press, Princeton, 1999). 
[8] M. Bunge, Twenty-five centuries of quantum physics: from Pythagoras to us, and fron subjectivism to realism, Sci. Educ. 12, 445 (2003).

[9] J. M. Sánchez Ron, El mundo después de la revolución (Pasado y presente, Barcelona, 2014).

[10] C. Passmore and J. Svodoba, Exploring opportunities for argumentation in modelling classrooms, Int. J. Sci. Educ. 34, 1535 (2012).

[11] R. Justi, La enseñanza de las ciencias basada en la elaboración de modelos, Enseñanza de las Ciencias 24, 173 (2006).

[12] I. T. Koponen, Models and modelling in physics education: a critical reanalysis of philosophical underpinnings and suggestions for revisions, Sci. Educ. 16, 751 (2007).

[13] P. S. Oh and S. J. Oh, What teachers of science need to know about models: an overview, Int. J. Sci. Educ. 33, 1109 (2011).

[14] J. R. Hoehn and N. D. Finkelstein, Students' flexible use of ontologies and the value of tentative reasoning: examples of conceptual understanding in three canonical topics of quantum mechanics, Phys. Rev. Phys. Educ. Res. 14, 010122 (2018).

[15] V. Sinarcas and J. Solbes, Dificultades en el aprendizaje y la enseñanza de la física cuántica en el bachillerato, Enseñanza de las Ciencias 31, 9 (2013).

[16] N. Didis and L. Wang, Students' mental models on atomic spectra, Chem. Educ. Res. Pract. 17, 743 (2016).

[17] N. Didis, A. Eryilmaz, and S. Erkoç, Investigating students' metal models about the quantization of light, energy and angular momentum, Phys. Rev. ST Phys. Educ. Res. 10, 020127 (2014).

[18] K. Krijtenburg-Lewrissa, H. J. Pol, A. Brinkman, and W. R. Jooligen, Insights into teaching quantum mechanics in secondary and lower undergraduate education, Phys. Rev. Phys. Educ. Res. 13, 010109 (2017).

[19] F. Savall-Alemany, J. L. Doménech, J. Guisasola, and J. Martínez-Torregrosa, Identifying student and teacher difficulties in interpreting atomic spectra using a quantum model of emission and absorption of radiation, Phys. Rev. Phys. Educ. Res. 12, 010132 (2016).

[20] L. Ivanjek, P. S. Shaffer, L. C. McDermott, M. Planinic, and D. Veza, Research as a guide for curriculum development: An example from introductory spectroscopy. I. Identifying student difficulties with atomic emission spectra, Am. J. Phys. 83, 85 (2015).

[21] C. Singh, Student understanding of quantum mechanics, Am. J. Phys. 69, 885 (2001).

[22] Link to the simulations: https://phet.colorado.edu/es/ simulations/category/physics/quantum-phenomena.

[23] L. Ivanjek, P. S. Shaffer, L. C. McDermott, M. Planinic, and D. Veza, Research as a guide for curriculum development: An example from introductory spectroscopy. II. Addressing students' difficulties with atomic emission spectra, Am. J. Phys. 83, 171 (2015).

[24] ROYAL DECREE 1467/2007, dated 2 November, establishing the structure of the baccalaureate and setting its basic syllabus. BOE (State Gazette) 6th November 2007. https://www.boe.es/boe/dias/2007/11/06/pdfs/ A45381-45477.pdf.
[25] H. Fischler and M. Lichfeld, Modern physics and students' conceptions, Int. J. Sci. Educ. 14, 181 (1992).

[26] M. Michelini, R. Ragazzon, L. Santi, and A. Stefanel, Proposal for quantum physics in secondary school, Phys. Educ. 35, 406 (2000).

[27] M. Budde, H. Niedderer, P. Scott, and J. Leach, 'Electronium': a quantum atomic teaching model, Phys. Educ. 37, 197 (2002).

[28] J. H. Wandersee, J. J. Mintzes, and J. D. Novak, Research on Alternative Conceptions in Science, Handbook of Research on Science Teaching and Learning (Macmillan Publishing Company, New York, 1994).

[29] M. Meheut and D. Psillos, Teaching-learning sequences: Aims and tools for science education research, Int. J. Sci. Educ. 26, 515 (2004).

[30] R. A. Duschl and R. J. Hamilton, Conceptual Change in Science and in the Learning of Science in International Handbook of Science Education, edited by B. J. Fraser and K. G. Tobin (Kluwer, London, 1998).

[31] E. Etnika, A. Karelina, and M. Ruibal-Villasenor, How long does it take? A study of student acquisition of scientific abilities, Phys. Rev. ST Phys. Educ. Res. 4, 020108 (2008).

[32] J. Schwab, The Teaching of Science as Inquiry, in The Teaching of Science edited by J. Schwab and P. Brandwein (Harvard University Press, Cambridge, MA, 1962).

[33] C. Becerra-Labra, A Gras-Martí, and J. MartínezTorregrosa, Effects of a problem-based structure of physics contents on conceptual learning and the ability to solve problems, Int. J. Sci. Educ. 34, 1235 (2012).

[34] J. Martínez Torregrosa, R. Limiñana, A. Menargues, and R. Colomer, In-depth teaching as oriented-research about seasons and the sun/earth model: Effects on content knowledge attained by pre-service primary teachers, J. Baltic Sci. Educ. 17, 97 (2018).

[35] J. Guisasola, K. Zuza, J. Ametller, and J. GutierrezBerraondo, Evaluating and redesigning teaching learning sequences at the introductory physics level, Phys. Rev. ST Phys. Educ. Res. 13, 020139 (2017).

[36] K. Zuza, J. M. Almudí, A. Leniz, and J. Guisasola, Addressing students' difficulties with Faraday's law: A guided problem solving approach, Phys. Rev. ST Phys. Educ. Res. 10, 010122 (2014).

[37] D. Gil-Pérez and J. Carrascosa-Alís, Bringing pupils' learning closer to a scientific construction of knowledge: a permanent feature in innovations in science teaching, Sci. Educ. 78, 301 (1994).

[38] D. Gil-Pérez, J. Guisasola, A. Moreno, A. Cachapuz, A. M. Pessoa de Carvalho, J. Martínez Torregrosa, J. Salinas, P. Valdés, E. González, A. Gené Duch, A. Dumas-careé, H. Tricárico, and R. Gallego, Defending constructivism in science education, Sci. Educ. 11, 557 (2002).

[39] P. Newton, R. Driver, and J. Osborne, The place of argumentation in the pedagogy of school science, Int. J. Sci. Educ. 21, 553 (1999).

[40] R. Driver, P. Newton, and J. Osborne, Establishing the norms of scientific argumentation in classrooms, Sci. Educ. 84, 287 (2000). 
[41] L. Osuna, J. Martínez Torregrosa, J. Carrascosa, and R. Verdú, Planificando la enseñanza problematizada: el ejemplo de la óptica geométrica en educación secundaria, Enseñanza de las Ciencias 25, 277 (2007).

[42] L. Osuna, J. Martínez Torregrosa, and A. Menargues, Evaluación de la enseñanza problematizada sobre la luz y la visión en la educación secundaria obligatoria, Enseñanza de las Ciencias 30, 295 (2012).

[43] J. Guisasola, J. M. Almudí, and K. Zuza, The design and evaluation of an instructional sequence on Ampere's law, Am. J. Phys. 78, 1207 (2010).

[44] J. Martínez Torregrosa, J. L. Doménech, A. Menargues, and G. Romo, La integración de los trabajos prácticos en la enseñanza de la química como investigación dirigida, Educación Química 23, 112 (2012).

[45] H. Kragh, Quantum Generations: A History of Physics in the Twentieth Century (Princeton University Press, Princeton, NJ, 2002).

[46] A. B. Arons, Teaching Introductory Physics (John Wiley and Sons, New York, 1997).

[47] G. Holton and S. G. Brush, Introduction to Concepts and Theories in Physical Science (Princeton University Press, Princeton, NJ, 1985).

[48] M. Jammer, The Conceptual Development of Quantum Mechanics (Mc Graw Hill, New York, 1966).

[49] J. Martínez Torregrosa, F. Savall, J. L. Doménech, A. Rey, and S. Rosa, La enseñanza problematizada de la física cuántica en el nivel introductorio. Una propuesta fundamentada, Revista de Enseñanza de la Física 28, 77 (2016).

[50] F. Savall, J. L. Doménech, and J. Martínez Torregrosa, La enseñanza problematizada de la física cuántica en el bachillerato y en los cursos introductorios de física, Mod. Sci. Educ. Learn. 10, 199 (2017).

[51] F. Savall-Alemany, J. L. Doménech-Blanco, and J. Martínez Torregrosa, El espectroscopio cuantitativo como instrumento para la construcción y uso de modelos de emisión y absorción de radiación en física cuántica, Revista Brasileira de Ensino de física 36, 4302 (2014).

[52] N. Didis, A. Eryilmaz, and S. Erkoç, The influence of instructional interactions on students' mental models about the quantization of physical observables: a modern physics course case, Eur. J. Phys. 37, 015701 (2016).
[53] G. Kalkanis, P. Hadzidaki, and D. Stavrou, An instructional model for a radical conceptual change towards quantum mechanics concepts, Sci. Educ. 87, 257 (2003).

[54] P. Hadzidaki, The Heisenberg microscope: a powerful instructional tool for promoting meta-cognitive and meta-scientific thinking on quantum mechanics and the "nature of science", Sci. Educ. 17, 613 (2008).

[55] N. Bohr, On the constitution of atoms and molecules, Philos. Mag. 26, 1 (1913).

[56] Link to the teacher's guide (English version): https://rua .ua.es/dspace/bitstream/10045/92227/1/quantum_physics .pdf.

[57] Link to the students' material: http://rua.ua.es/dspace/ bitstream/10045/60628/1/cuantica_alumno.pdf.

[58] Link to the teacher's guide (Spanish version): http://rua .ua.es/dspace/bitstream/10045/60627/1/cuantica_profesor .pdf.

[59] Similar, for example, to J. D. Wilson and J. A. Buffa, College Physics, 3rd ed. (Prentice Hall, Englewood Cliffs, NJ, 1997).

[60] S. Kvale and S. Brinkmann, InterViews: Learning the Craft of Qualitative Research Interviewing, 2nd ed. (Sage Publications Inc., Thousand Oaks, CA, 2009).

[61] S. Kvale, Interviews. An Introduction to Qualitative Research Interviewing (Sage, Thousand Oaks, CA, 1996).

[62] L. Cohen, L. Manion, and K. Morrison, Research Methods in Education (Routledge/Falmer, London, 2000).

[63] J. Cohen, Statistical Power Analysis for Behavioural Sciences (Academic Press, New York, 1998).

[64] J. Petri and H. Niedderer, A learning pathway in highschool level quantum atomic physics, Int. J. Sci. Educ. 20, 1075 (1998).

[65] K. S. Taber, When the analogy breaks down: modelling the atom on the solar system, Phys. Educ. 36, 222 (2001).

[66] M. Budde, H. Niedderer, P. Scott, and J. Leach, The quantum atomic model 'Electronium': a successful teaching tool, Phys. Educ. 37, 204 (2002).

[67] G. Kalkanis, P. Hadzidaki, and D. Stavrou, An instructional model for a radical conceptual change towards quantum mechanics concepts, Sci. Educ. 87, 257 (2003).

[68] S. Sidiqui and C. Singh, How diverse are physics instructors' attitudes and approaches to teaching undergraduate level quantum mechanics?, Eur. J. Phys. 38, 035703 (2017). 